\title{
Similitude et cavitation ${ }^{(*)}$
}

PAR

\author{
J.M. ROBERTSON (**), J.H. MCGINLEY(***) IT J.W. HOLL (****) \\ ORDNANCE RESEARCH IABOHATORY, THE PENNSYIVANIA STATE INIVERSITY, VNIVERSTTY PARK, PENNSYLVANIA
}

(English text p. 550)

Les expériences sur lapparition de la cavita-
lion entreprises par les auteurs sur des corps
'ylindriques circulares abec nez en ogive, de
calibre variant entre o et 1,5 , montrent que le
fruteur dominant dont dépend le seuil de cette
apparition est la courbure de la surface, ou le
calibre. Mais les propriétés des fluides soumis
a l'examen, les dimensions des objets et les
vitesses d'essais influencent également le phé-
nomène. Les auteurs montrent que sur des nez
fins, l'apparition de la cavitation est surtout
subordonnée au produit de la vitesse par la
racine sarrée du diamètre du corps, tandis que,

\section{Introduction}

Maints problèmes de dynamique des fluides sont traités à échelle réduite, dans des tunnels aérodynamiques et hydrodynamiques, aux bassins de carène, etc., l'étude sur l'objet réel étant hérissée de difficultés. Pour bien interpréter les résultats des études sur modèle, il faut commencer par définir convenablement les paramìtres de similitude caractérisant les écoulements et déterminer les lois de similitude ou de transposition qui permettent de passer du modèle au prototype. Dans le cas des fluides incompressibles avec frottement, le nombre de Reynolds est considéré comme le paramètre approprié et on y rapporte les expressions concernant la couche limite et la traînée. S'il s'agit de fluides compressibles, c'est le nombre de Mach qui compte, et pour les liquides à surface libre, le nombre de Froude. Pour les liquides en écoulement lent à surface libre, la tension superficielle jouant un

(*) Etude réalisée pour le compte du U. S. Navy Bureau of Ordnance (marché $\mathrm{n}^{\circ} 7958$ ).

(*) Précédemment Directeur adjoint Service "Water Tunnel », actuellement Professeur de mécanique théorique et appliquée à l'Université de l'Illinois.

(**) Précédemment Attaché de Recherches, actuellement auprès de Haller, Raymond \& Brown, Inc., State College, Pennsylvania.

$\left({ }^{* * *}\right)$ Attaché de Recherches.

\begin{abstract}
pour les formes obtuses, elle se rattache an nombre de Reynolds. Aux courbures intermé(diaires, par exemple anec le calibre $1 / 8$, les deux règles s'appliquent, et l'on peut obtenir différents coefficients critiques d'apparition. Le mémoire traite également le cas de deux régimes d'apparitión de la cavitation sur deux corps de formes différentes. L'extrapolation des résultats des essais peut donc, devoir s'effectuer selon deur lois différentes et l'apparition des deux régimes de cavitation pent correspondre à des coefficients critiques nettement dissemblables.
\end{abstract}

rôle le nombre de Weber intervient. Mais en ce qui concerne les écoulements avec cavitation, la situation n'est pas encore bien claire.

Le paramètre sans dimension décrivant le mieux la nature d'un écoulement cavitant est ce qu'on appelle « le coefficient de cavitation »:

$$
\sigma=\frac{P-P_{r}}{1 / 2 V^{\prime 2}}
$$

P désignant la pression statique absolue dans l'écoulement, $\mathrm{P}_{\mathrm{Y}}$ la pression de vapeur du liquide, $?$ la densité de masse et $\mathrm{V}$ la vitesse d'écoulement libre, c'est-à-dire la vitesse relative du corps par rapport au fluide supposé non troublé. La valeur de ce nombre lors de la toute première apparition de la cavitation est désignée par « coefficient critique ou d'apparition».

Dans un écoulement avec cavitation, le coefficient $\sigma$ a une valeur inférieure à la valeur d'apparition $\sigma_{c}$ relative au corps considéré, la cavitation étant d'autant plus développée que la valeur de $\sigma$ est plus faible que $\sigma_{c}$. Dans l'ćlude de la cavitation, on admet souvent que lindice d'apparition $\sigma_{c}$ est fonction uniquement de la forme du corps et de son orientation au scin du fluide, mais ce n'est là qu'une toule première approximation; certaines autres conditions concernant le fluide interviennent également. 
Kermeen, McGraw et Parkin [1] du Laboratoire d'Hydrodynamique de l'Institut Technologique de Californie, ont récemment publié les résultats de leurs recherches concernant l'apparition de la cavitation pour des corps présentant une symétrie axiale. Les résultats auxquels ils sont parvenus montrent que les lois de similitude diffèrent selon qu'il s'agit de corps profilés ou non. Dans le premier cas, celui des corps cylindriques à nez en forme d'ogive, de calibre 0,5 et 1,5 , le coefficient d'apparition de la cavitation augmente avec les dimensions du modèle et avec la ritesse de l'écoulement libre et tend à devenir égal au coefficient de pression minimum. S'il s'agit de disques à arête vive, cas extrême du corps non profilé, le coefficient critique semble varier avec le nombre de Reynolds.

Par contre, l'analyse des effets de la variation de la vitesse et des dimensions des corps de forme profilée étudiés a montré que le coefficient critique dépend de $\mathrm{V} d^{1 / 2}$ (produit de la vitesse par la racine carrée du diamètre du corps), c'est-à-dire du nombre de Weber [2]. Ainsi, les lois qui gouvernent la similitude de la cavitation varieraient avec la forme du corps. En pratique, le problème consiste à déterminer quelle loi devra être appliquée pour transposer au prototype les résultats d'essais sur modèle d'un corps ou objet de forme donnée. Des études récentes, effectuées au tunnel hydrodynamique de $122 \mathrm{~cm}$ du « Laboratoire de Recherches sur les Armements »(ORL) relevant de l'Université de l'Etat de Pennsylvanie, ont permis d'éclairer la question, mais il n'est pas encore possible d'en brosser un tableau d'ensemble.

\section{Essais du tunnel hydrodynamique}

La cavitation a été étudiée au tunnel hydrodynamique ORL sur des disques et sur des nez de différents aspects. Le cas extrême du corps non profilé a été réalisé à l'aide d'un cylindre circulaire droit placé parallèlement à l'écoulement; ce cylindre, avec son extrémité plate, peut être considéré comme une ogive de calibre zéro, par conséquent trouver sa place dans la famille des ogives d'aspect aérodynamique. Les caractéristiques de l'apparition de la cavitation pour une telle famille d'ogives ont fait l'objet d'études extrêmement poussées [3] [4]. La figure 1 montre les différents contours expérimentés $\left(^{\star}\right)$. Les nez de calibre $0,1 / 8,1 / 4$ et $1 / 2$ et de diamètre $1 / 2,1,2,3$ et 4 pouces ont été étudiés au tunnel hydrodynamique de diamètre intérieur $122 \mathrm{~cm}$, à des vitesses variant

(*) Les formes des calibres 0 à $1 / 2$ sront sourent désigués par " ogives plates $»$. entre 6 et $24 \mathrm{~m} / \mathrm{s}$. Aux essais en Lunnel décrits à l'ourvage réf. [2] et dont nous citerons quelques-uns des résultats, des nez de $1 / 4^{\prime \prime}$ à $8^{\prime}$

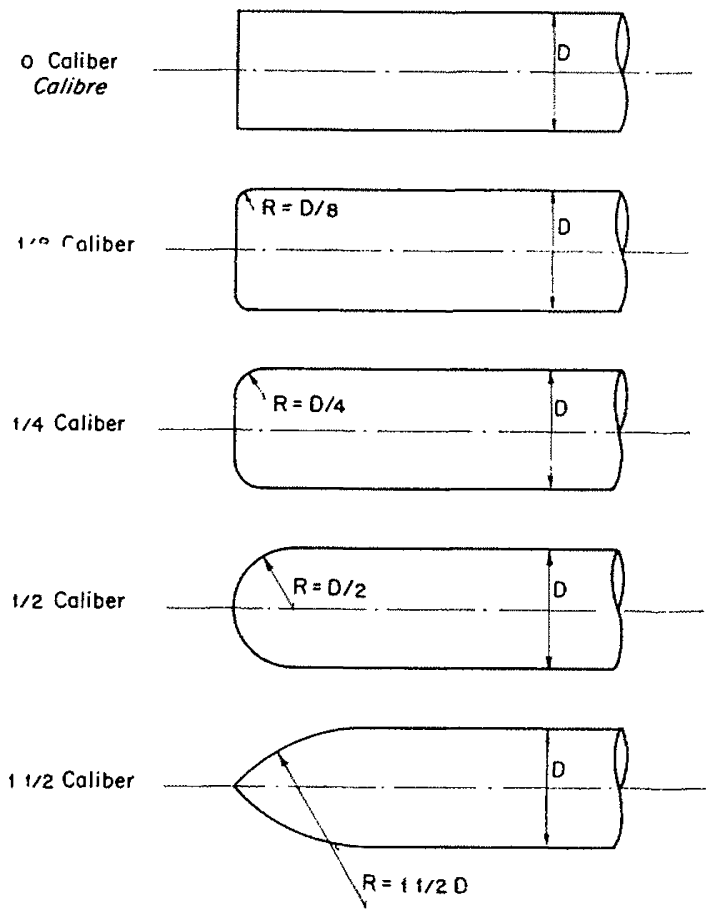

Fic. 1. - Formes d'ogives.

Ogive nose contours.

$(0,64$ à $20,32 \mathrm{~cm})$ de calibre $1 / 2$ el $11 / 2$ ont été expérimentés.

Mais pour avoir des valeurs reproductibles, et éliminer tout effet "d'hystérésis", nous avons déterminé les conditions d'apparition de la cavitation selon le procédé utilisé au « Caltech »



Fig. 2. - Aspect caractéristique de l'apparilion acoustique de la cavifation.

Typical acoustic catuitation inception conditions. 
(Institut Technologique de Californie) [1] [2], c'est-à-dire que pour une certaine vitesse de l'écoulement, on abaissait la pression jusqu'à l'apparition de la cavitation, puis on la relevait jusqu'à ce que la cavitation eût disparu. La disparition des derniers vestiges de cavitation se laisse d'ordinaire saisir à l'œil nu; toutefois pour la plupart de nos observations, nous nous sommes servis d'un dispositif acoustique hydrophonique sensible aux ultra-sons [5], grâce auquel l'apparition de la cavitation produit généralement de fortes variations de la sonorité; un enregistreur automatique du niveau sonore donne alors des diagrammes semblables à celui de la figure 2, sur lesquels on distingue nettement la pression d'apparition du phénomène. Très souvent, l'observation visuelle fournissait une pression critique de cavitation s'écartant de $0,2 \mathrm{psi}\left(0,014 \mathrm{~kg} / \mathrm{cm}^{2}\right)$ de celle obtenue par voie acoustique. La pression minimum utilisée dans le tunnel pendant ces essais était de 5,5 psi $\left(0,39 \mathrm{~kg} / \mathrm{cm}^{2}\right)$ donnant pour l'indice de cavitation, une erreur maximum possible de $4 \%$ environ.

L'interprétation des résultats des essais était basée sur un coefficient critique de cavitation déduit de la définition précitée, la pression de vapeur étant déterminée d'après la température de l'eau pendant l'essai. Le nombre de Reynolds $\mathcal{R}=V d / \nu$ a été exprimé en fonction du diamètre du corps cylindrique auquel les nez appartenaient, la température de l'eau mesurée au cours des essais servant à déterminer la viscosité cinématique v. Bien que prévenus du fait que l'apparition de la cavitation pourvait dépendre du nombre de Weber (donc de l'apparition d'effets de tension aux surfaces), nous avons considéré les variations de la tension superficielle avec de l'eau ordinaire comme trop faibles pour faire l'objet d'une étude spéciale et nous n'avons considéré que le facteur dimensionnel $\mathrm{Vd}^{1 / 2}$.

\section{L'apparition}

\section{de la cavitation sur nez non profilé}

Les résultats obtenus à Caltech sur des disques [1] témoignent d'une forte sujétion au nombre de Reynolds. La cavitation observée sur les cylindres à extrémité aplatie étant très semblable à celle que l'on constate sur les disques $\left(^{*}\right)$, on pourvait en déduire qu'ici encore l'influence du nombre de Reynolds se ferait sentir. C'est ce qui a été trouvé, comme on le voit sur les valeurs données par la figure 3 , pour des nez de $1 / 2,1,2$ et 3 pouces $(1,28-2,54-5,08$

(*) Du moins près de l’apparition. et $7,62 \mathrm{~cm}$ ). Abstraction faite de la dispersion, qui est du même ordre que pour les valeurs de Caltech sur disques, la concordance est assez

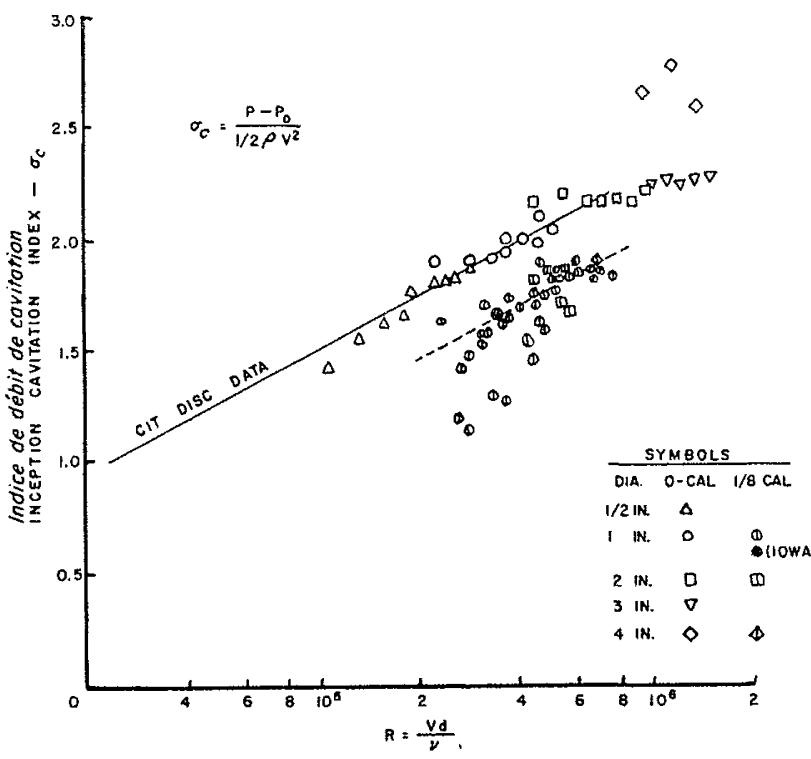

Fig. 3. - Influence du nombre de Reynolds sur la cavitation du type à décollement.

Reynolds number scaling of separation type cavitation.

bomne avec la courbe relative aux disques. Par contre, les valeurs obtenues pour l'ogive de calibre zéro ne dépendent pas de $\mathrm{V} d^{1 / 2}$ puisque le tracé obtenu en fonction de ce facteur conduit $\dot{a}$ des courbes distinctes pour les différentes dimensions.

L'apparition de la cavitation sur ogive de 4 pouces $(10,65 \mathrm{~cm})$, calibre zéro, a fourni des valeurs, d'ailleurs peu nombreuses et compara-

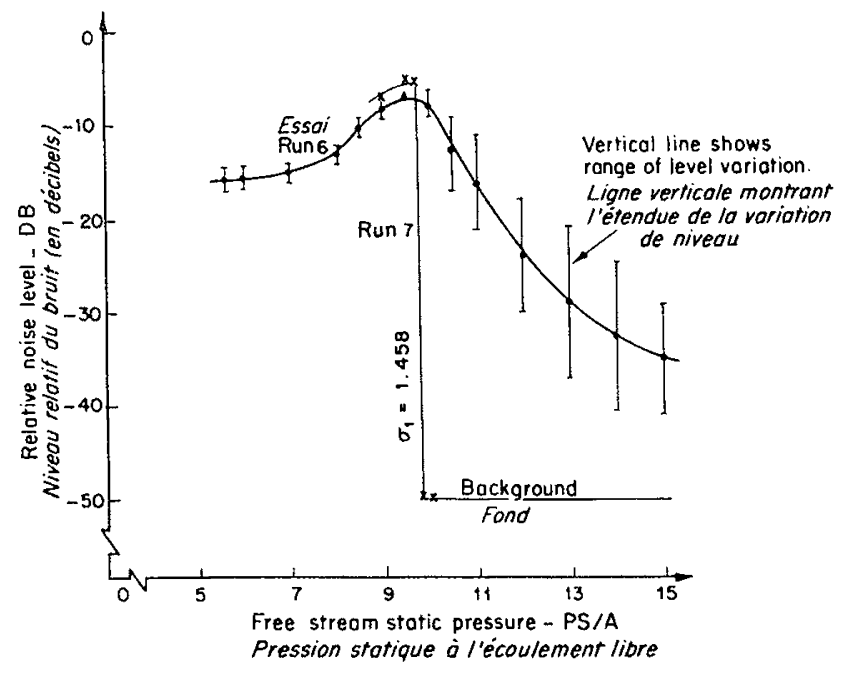

Fic. 4. - Effet des bulles d'air sur la détection acoustique de l'apparition de la cavitation.

Effect of air bubbles on acoustic determination of cuvitalion inception. 
tivement diflérentes de celles qui ont élé oblenues sur des corps moins grands. Il semble bien que ceci soit dû à des phénomènes de dégaģement d'air ayant troublé quelques-uns des derniers essais, surtout aux faibles vitesses. On ne pouvait songer à étudier l'extrémité large à des vitesses suffisamment grandes pour noter la variation subie par le coefficient critique de cavitation et pour se rendre compte exactement de ce qui se passait. Cependant, nous avons pu noter de façon claire l'influence d'un dégagement d'air sur l'apparition de la cavitation dans le cas d'une ogive de 1 pouce $(2,54 \mathrm{~cm})$, calibre $1 / 8$. Lal figure 4 montre les enregistrements acoustiques obtenus pour deux séries d'essais dans des conditions identiques de courant moyen. En moyenne, la teneur en air élait élevée, et l'on a pu observer pendant l'essai initial $\left(n^{\circ} 6\right)$ la formation de petites bulles d'air à l'approche du nez; dans ce cas, l'apparition de la cavitation ne se marque pas sur l'enregistrement d'une façon aussi nette que dans le cas de la figure 2. Afin d'éliminer les bulles d'air, on a fait fonctionner d'abord le tunnel sous pression relativement élevée pendant quelques minutes, puis on a recommencé l'essai rapidement avant que de nouvelles bulles n'aient pu se former; pendant cette nouvelle reprise (essai 7) la cavitation est apparue de façon normale. Comme le volume total de l'air présent dans le tunnel restait le même au cours des essais, on peut penser que les petites bulles formées lors du premier essai, voilaient la véritable apparition de la cavitation. Nous dépasserions les limites de cette étude si nous cherchions à décider lequel des deux cas est le plus proche de la réalité.

A en juger d'après les valeurs obtenues avec l'ogive de calibre $1 / 8$, le corps en question se comporterait comme s'il n'était pas profilé. Sur la figure 3 , les valeurs correspondant à ce cas pour les diamètres de 1,2 et 4 pouces ont donc été portées en fonction du nombre de Reynolds, en $y$ ajoutant un point provenant d'essais sur un nez de 1 pouce effectués à l'Université d'Iowa [3]. Sauf pour certains points bas-silués, dus à des essais sur corps de 1 pouce, les valeurs caractérisant les ogives de calibre $1 / 8$ indiquent une variation du coefficient d'apparition de la cavitation en fonction du nombre de Reynolds semblable à celles qui marquent les extrémités de calibre zéro. Nous verrons par la suite que l'écoulement dans ce cas était de nature très différente de celui qui apparaissait quand une variation en fonction de $V d^{1 / 2}$ semblait intéressante; d'autre part, le coefficient critiçue d'apparition avait alors une valeur bien moins forte que lors d'ćcoulements d'un autre genre, pour lespuels une similitude basée sur le facteur $\mathrm{V} d^{1 / 2}$ semblait à retenir.

\section{Apparition \\ de la cavitation sur nez de forme profilée}

Comme il a déjà été dit, le paramètre $V d^{1 / 2}$ pouvait permettre d'établir une relation entre l'apparition de la cavitation sur les ogives de calibre 0,5 et 1,5 [2]. La figure 5 donne, en fonction de $V d^{1 / 2}$, les valeurs d'apparition du phé-

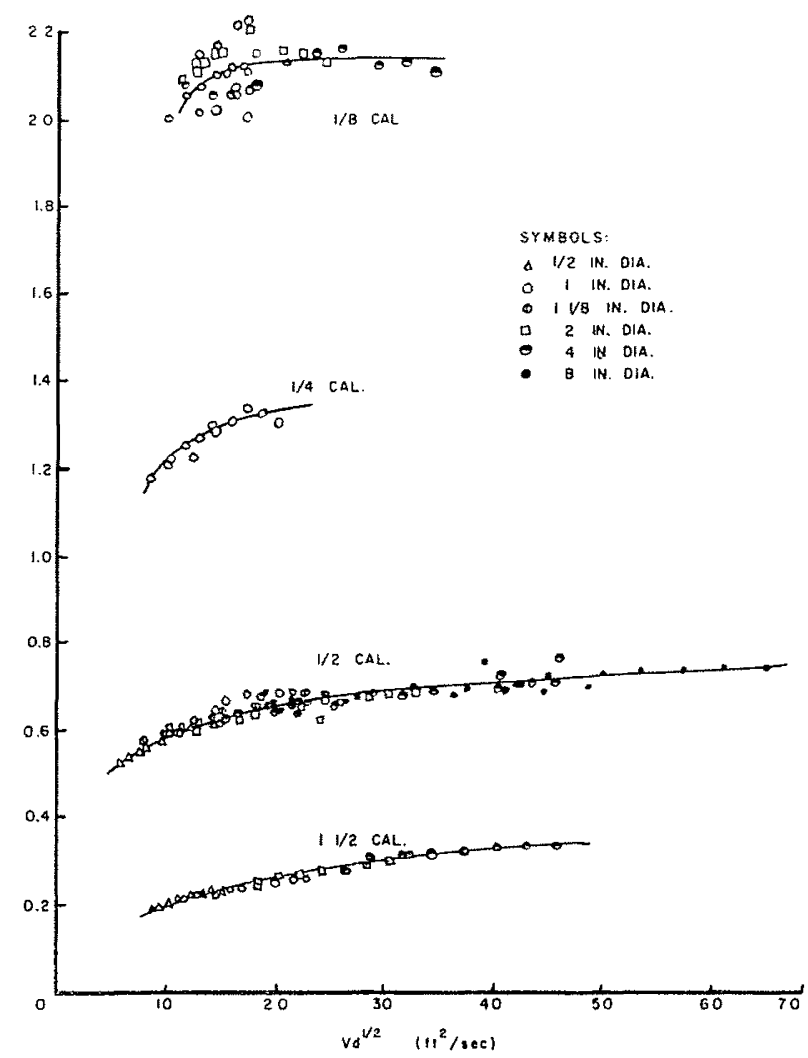

FIf. 5. - Variation de la cavitation selon Vd1/2.

Cavitation scaling with $\mathrm{V} d 1 / 2$.

nomène obtenues à l'ORL (Laboratoire de Recherches sur les Armements) pour les deux formes de corps signalées. Elle comprend, en dehors des valeurs ORL tirées de l'ouvrage réf. [2] pour des ogives variant de 0,5 à 8 pouces, une série de mesures plus récentes sur un nez de 1 pouce, calibre 0,5. La concordance en fonction de $\mathrm{Vd}^{1 / 2}$ est manifeste comme l'indique d'ailleurs la réf. [2] qui prouve également que la relation basée sur le nombre de Reynolds n'est vraiment pas très marquée. Remarquons que la même référence montre que les valeurs du Caltech (non portées sur la figure 5), relatives aux nez de $1 / 4$ à 4 pouces $(0,64$ à $10,15 \mathrm{~cm})$ des deux mêmes calibres, concordent exactement avec les valeurs ORL; de fait, le plus srand ćarl entre l'allure des courbes représentant les valeurs du coefficient d'apparition obtenues aux deux tunnels est d'environ 0,02 pour un $V d^{1 / 2}$ voisin de 20 . Enfin, 
on a pu constater que pour les deux formes étudiées (calibres 0,5 et 1,5 ), le coefficient critique de cavitation est inférieur au coefficient de pression minimum du corps, mais qu'il s'en rapproche à mesure qu'augmentent les dimensions et la vitesse [1] [2].

Sur la figure 5 ont également été portées les valeurs obtenues aux essais sur ogives non profilées (calibres $1 / 4$ et $1 / 8$ ). Le cas du calibre $1 / 4$ n'ayant été étudié que pour un diamètre de 1 pouce, on ne peut apprécier la valeur de la concordance en fonction de $\mathrm{Vd}^{1 / 2}$. Certaines valeurs de la même figure laissent entrevoir la relation à $V^{1 / 1 / 2}$ dans le cas de l'ogive calibre $1 / 8$; cependant, la dispersion est ici bien plus forte que pour les ogives profilées, bien que la concordance soit assez bonne pour les dimensions 1,2 et 4 pouces.

\section{Influence du calibre sur le coefficient critique}

Selon les essais effectués à l'Université de l'Etat d'Iowa [3], rapportés par Rouse et McNown en 1948, il existe une relation unique entre le coefficient critique d'apparition de la cavitation et le calibre, pour les extrémités rondes qu'ils ont étudiées, c'est-à-dire des nez de 1 pouce à des vitesses atteignant $35 \mathrm{fps}(10,5$ $\mathrm{m} / \mathrm{s}$ ). Pour définir l'apparition du phénomène, les auteurs s'étaient alors basés sur la répartition des pressions, selon un procédé qui tire parti du fait que la cavitation altère la répartition des pressions sur la surface (pressions relevées sous forme d'un coefficient de cavitation). Le coefficient critique $\sigma_{\theta}$ était alors choisi comme celui qui correspondait à la perturbation initiale de cette répartition. Les valeurs trouvées à Iowa, établissant la relation entre le coefficient d'apparition de la cavitation et le calibre, ont souvent servi à des études hydrobalistiques. Cependant une question restait ouverte : celle de savoir si, en se basant sur d'autres critères (visuels, acoustiques) d'apparition de la cavitation, on n'obtiendrait pas des valeurs nettement différentes pour le coefficient critique; cette incertitude peut conduire à un choix trop généreux des « coefficients de sécurité », voire même introduire des erreurs dans les calculs. La publication concernant les travaux d'Iowa comprend également certaines valeurs Caltech [4], obtenues par observation visuelle de la poche de vapeur qui se forme au début du phénomène, et qui, dans le cas de calibres inférieurs à 2 , indiquaient des valeurs de $\sigma_{c}$ nettement plus faibles.

Nos propres recherches permettent d'élablir la comparaison entre les conditions qui régissent l'apparition de la cavitation relevées par voie acoustique et les conditions qu'indique le pro- cédé des pressions. Cetle comparaison des différents $\sigma_{c}$ en fonction du calibre est représentée sur la figure 6 , où les valeurs d'Iowa figurent sous la forme d'une courbe unique, tandis que

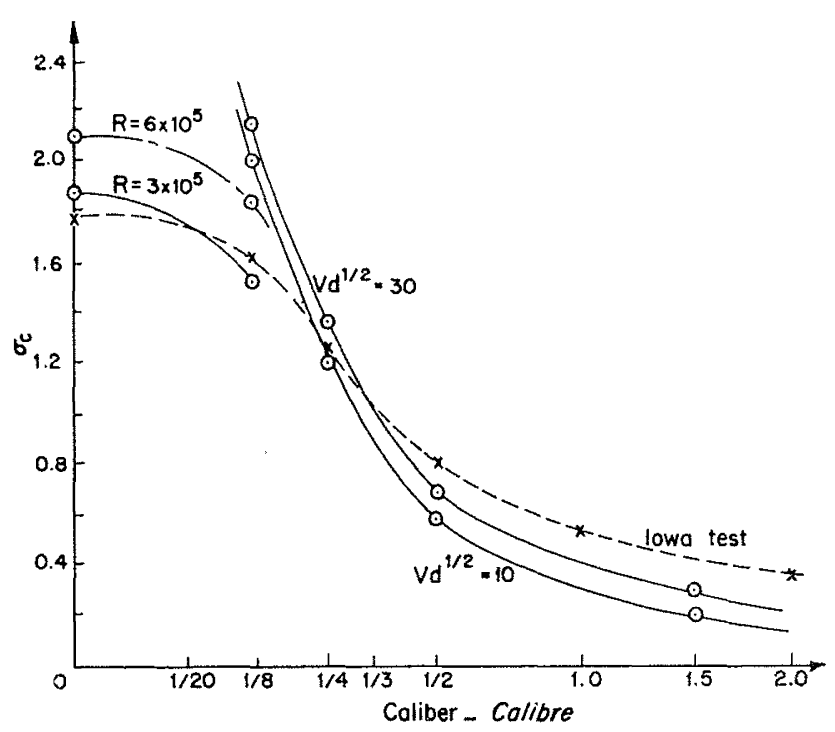

Fis, 6. - Variation du coefficient critique de cavitation avec le calibre pour des ogives.

Cabilation inception index nariation with caliber for ogive noses.

les nôtres fournissent quatre tracés différents. On $y$ trouve en effet les courbes correspondant à un $\mathrm{Vd}^{1 / 2}$ égal à 10 et à 30 , pour les cas où le rapport à ce paramètre était possible; ces deux valeurs ayant été retenues parce que, comme le montre la figure 5 , le $\sigma_{c}$ augmente très lentement quand $V d^{1 / 2}$ devient supérieur à 30 , tandis que la valeur 10 de ce paramètre représente la limite inférieure de la confiance que méritent les essais; cette même valeur de 10 correspond d'ailleurs à la limite supérieure des essais d'Iowa (nez de 1 pouce à la vitesse de $35 \mathrm{fps}=$ $10,5 \mathrm{~m} / \mathrm{s}$ ). Pour des nez auxquels s'appliquent la relation du nombre de Reynolds, on a les deux courbes $R=3 \times 10^{5}$ el $\mathscr{R}=6 \times 10^{\circ}$. Nous ignorons les nombres de Reynolds précis pour lesquels ont été obtenues les valeurs d'lowa des ogives non profilées, mais, selon les informations recues, quelques-uns de leurs essais étaient du domaine $2 \times 10^{5}$ à $6 \times 10^{5}$.

La figure 6 montre le rôle prédominant que joue la forme, c'est-à-dire le calibre, dans la détermination de l'apparition de la cavitation. On y constate igalement l'importance des deux paramètres qui gouvernent la similitude du phénomène. Dans le cas des calibres dépassant $1 / 3$, la méthode de détermination de $\sigma_{r}$ par la pression attribue au coefficient critique des valeurs fortes. D'autre part, pour les calibres inférieur's, le bruil causé par la cavitation apparait d'ordinaire avant que la cavitation ne devienne assez 


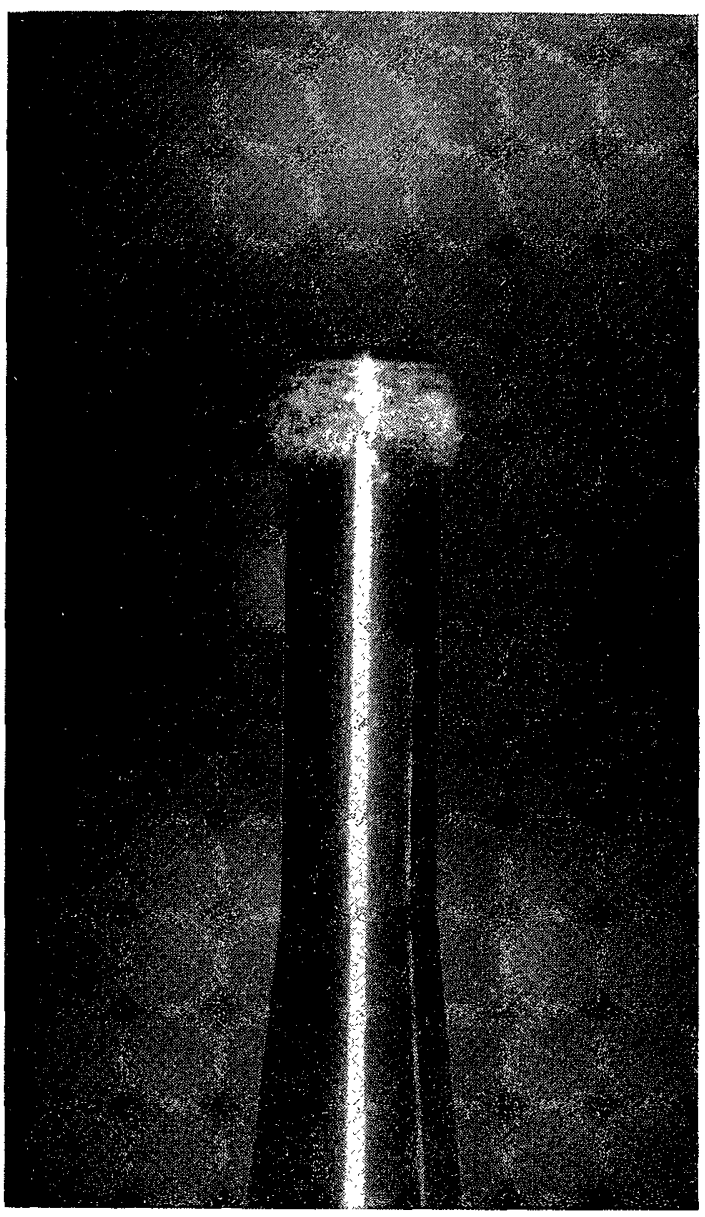

Frg. 7 a. - Cavitation avec décollement.

Separation type cavitation.

forte pour modifier la répartition des pressions. Avec les formes non profilées, le coefficient critique varie avec le nombre de Reynolds et les valeurs obtenues pour les pressions à Iowa semblent concorder avec celles obtenues par nous.

Kermeen, McGraw et Parkin [1] ont cherché une explication aux valeurs élevées que la méthode des pressions attribue à $\sigma_{c}$; ils ont constaté que la cavitation s'installe prématurément aux points de prélèvements de la pression, à cause de la nature de l'écoulement devant l'ouverture; ainsi, le procédé des pressions fournirait une indication quant à l'instant d'apparition de la cavitation en ces points, plutôt que sur le corps même. Les valeurs obtenues à Caltech, hasées sur une détermination visuelle de l'apparition du phénomène, et dont il a été fait état en 1945 [4], sont situées sur la figure 6 entre la courbe d'lowa et celle correspondant à $V^{r} d^{1 / 2}=30$, pour les calibres compris entre 0,5 et 2 ; dans le cas du calibre 1 , elles se rapprochent davantage des valeurs acoustiques que nous avons obtenues, puisqu'elles coïncident avec la courbe $V d^{1 / 2}=30$. Comme nous l'avons signalé précédemment, nous avons obtenu une concordance étroite entre nos valeurs visuelles et acoustiques et tout écart dans la détermination de l'apparition de la cavitation serait problement imputable aux difficultés d'éclairage et d'observation.

\section{Cavitation sur des extrémités de calibre 1/8}

Pour les différentes formes de pointes qu'ils ont étudiées, Rouse et $\mathrm{McNown}$ [3] n'ont trouvé des écoulements instables que pour des extrémités de calibre $1 / 8$ et pour un ellipsoide. Ils ont constaté dans les deux cas que l'écoulement tendait alternativement soit à prendre l'aspect irrotationnel, soit à former une poche de séparation, petite mais bien définie. Aussi bien les formes plus courtes que les formes plus allongées donnaient un régime stable pour des domaines égaux ou inférieurs à ceux du tunnel, mais pour des raisons différentes : l'écoulement rasant les formes courtes et concaves crée un effet de décollement stable partant du point de brusque transition du contour; tandis que sur les formes allongées, ou bien il n'y a pas de



Frs. $7 b$. - Cavitation de surface

Surface type cavitation. 
décollement, ou bien celui-ci est éliminé par la turbulence pour des valeurs de $\mathcal{R}$ relativement faibles. Pour les formes intermédiaires instables, à mesure que croît $\mathcal{R}$, le décollement est moins net et la valeur de la pression minimum est réduite; on arrive bien à une valeur limite, mais uniquement aux valeurs supérieures de $\mathcal{R}$ [3a].

Au cours de nos essais, nous avons constaté une instabilité semblable avec des nez de calibre $1 / 8$, comme l'indiquent les deux groupes de points d'apparition sur la figure 6. Le coefficient critique le plus fort se présentait ordinairement aux vitesses supérieures avec le même type de cavitation que celui noté pour les nez de calibre $1 / 4,1 / 2$ et $1 / 5$. Des vues photographiques des deux genres d'écoulement sont représentées à la figure $7 a$, qui illustre le cas du décollement du courant, et à la figure $7 b$ correspondant à une cavitation de surface.

Dans le premier cas, la cavitation se manifeste par des tourbillons dans la région de décollement du courant, exactement comme sur les ogives de calibre zéro, plús obtuses. Selon le second mode, elle se manifeste sous forme d'anncau fin sur la surface du nez, près du point de tangence entre la courbe de l'ogive et le corps auquel elle se raccorde.

Fréquemment, au cours de ces expériences, il a été possible d'observer visuellement les différences entre l'écoulement avec cavitation du type décollement et l'écoulement avec cavitation annulaire, mais ce n'était pas toujours le cas et, très souvent, les points d'apparition semblent avoir été mal identifiés. L'existence des deux relations de base de similitude, que prouvent les figures 3 et 5 en ce qui concerne les ogives de calibres $1 / 8$, a pu être nettement établie dans les essais pour lesquels le genre de cavitation pouvait ètre exactement identifié. On constatait alors que dans le cas de la cavitation annulaire $\sigma_{o}$ se plaçait entre 2,0 et 2,2 , tandis que la cavitation comportant un décollement lui attribuait des valeurs inférieures à 1,9. C'est en définitive d'après la valeur numérique du coefficient critique qu'était défini le genre de cavitation apparu.

Dans maints essais, le passage d'un genre d'écoulement à l'autre se manifestait entre essais réalisés à différentes vitesses; dans quelques autres, les deux genres se présentaient sous un même essai et à une même vitesse : à mesure qu'on élevait la pression, la cavitation genre dćcollement tendait à disparaître, ayant au passage indiqué son coefficient d'apparition, puis la cavitation annulaire s'installait, probablement causée par certaine fluctuation de moindre importance de l'écoulement; l'on avait dû alors

(3 a) H. Rouse et J. S. McNown [3] p. 13. surélever la pression pour établir les conditions d'apparition de ce nouveau type. La figure 8 se rapporte à quelques-uns des essais réalisés à des vitesses différentes sur des nez de 1 , de 2 , et de 4

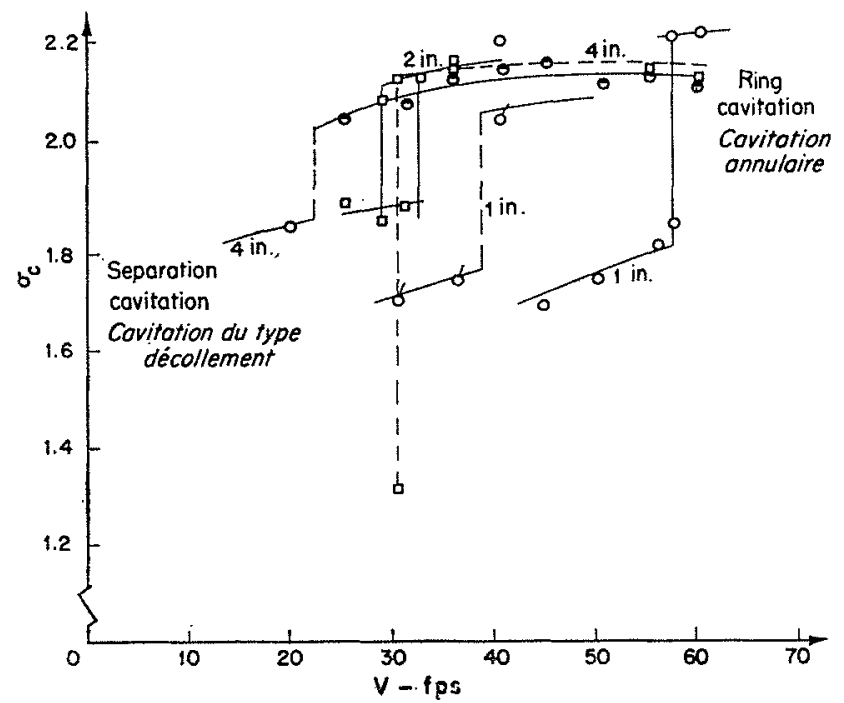

Fig. 8. - Manifestation de deux régimes d'apparition de la cavitation dans plusieurs séries d'essais.

Occurence of two regimes of cavitation inception for several series of tests.

pouces $(2,54,5,08$ et $10,16 \mathrm{~cm})$; les traits pleins verticaux concernent les cas où les deux types de cavitation ont pu être observés pour les mêmes conditions d'essai.

Selon l'avis de Rouse et McNown [3], nous avons supposé que la distinction entre les deux genres d'écoulement pouvait être basée sur le nombre de Reynolds. Cherchant à éclaircir ce

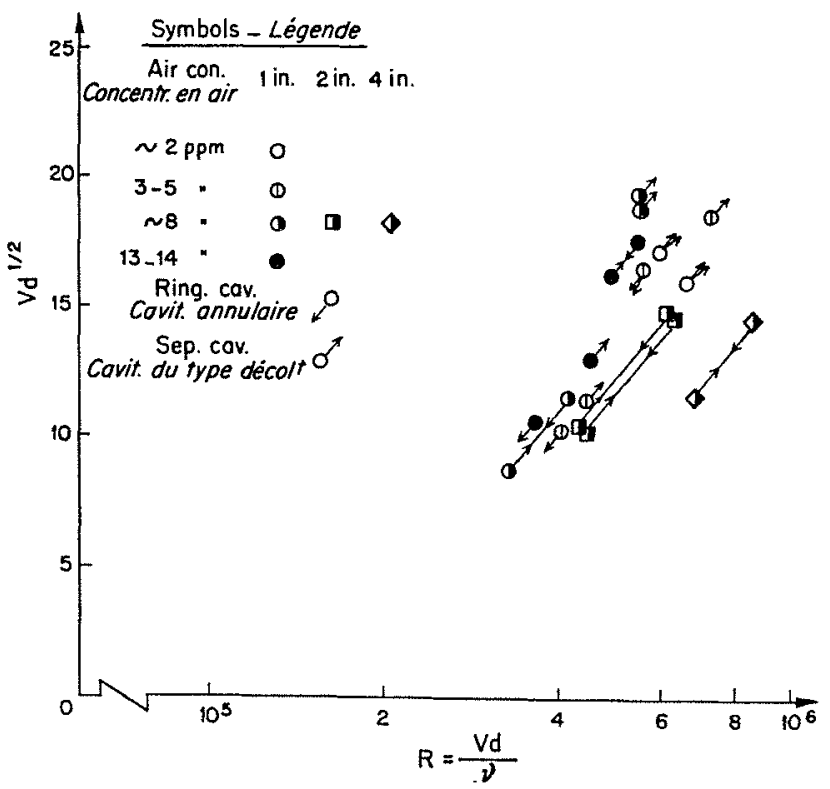

Fig. 9. - Démarcation entre deux régimes d'apparition de la cavitation.

Demarkation between two regimes of cavitation inception. 
point, nous avons effectué un grand nombre d'essais sur des nez de 1 pouce, la température de l'eau variant entre 77 el $107^{\circ} \mathrm{F}$ ( 25 et $42^{\circ} \mathrm{C}$ ). Nous n'avons pas essayé de doser de façon absolue l'air présent dans l'eau; sa proportion variait depuis environ 2 jusqu'à 14 millionnièmes, au cours de cette série d'essais. Il nous est arrivé de ne rencontrer qu'un seul genre d'écoulement; parfois l'essai n'avait pas été conduit à une vitesse suffisante ou, au contraire, celle-ci n'était pas suffisamment réduite. Les valeurs obtenues pendant cette phase de notre recherche sont données sur la figure 9. Souvent les essais n'ont mis en évidence qu'un seul genre de cavitation. En admettant que la cavitation annulaire a lieu à des vitesses plus grandes que celle qui se caractérise par un décollement, les flèches adjointes aux points représentatifs indiquent la direction dans laquelle l'autre genre de cavitation est censé se produire. 'Tout ce qu'on peut déduire de cette étude, c'est que la distinction entre la cavitation annulaire et la cavitation avec décollement, sur des ogives de calibre $1 / 8$, se manifeste pour des nombres de Reynolds compris entre $3 \times 10^{5}$ et $8 \times 10^{5}$, et pour de valeurs de $\mathrm{V} d^{1 / 2}$ comprises entre 10 et 20 . On ne saurait affirmer que la teneur en air $y$ est pour quelque chose.

Avant de clore la discussion, il convient de noter que la courbe représentant $\sigma_{c}$ en fonction de $\mathcal{O}$ s'approche de la valeur de $\sigma_{c}$ qui correspond à la cavitation genre annulaire pour un certain nombre de Reynolds supérieur à $10^{6}$; audessus, la distinction entre les deux genres de cavitation semble toute théorique.

\section{Présence des deux régimes de cavitation sur d'autres corps}

En dehors de l'ogive de calibre $1 / 8$ et du nez ellipsoïdal 1/2 : 1 qui ont été étudiés par Rouse et McNown, deux autres formes expérimentées au tunnel hydrodynamique de l'ORL ont présenté les deux régimes de cavitation, entraînant deux lois de similitude possibles pour un même corps.

Le premier cas concerne une partie du tunnel même. Pour accéder à la section de travail, de 4 pieds $(1,22 \mathrm{~m})$ de diamètre, du tunnel, il faut écarter un volet amovible faisant partie de la surface supérieure de la partie cylindrique [5]; ce volet doit évidemment être très exactement adapté au cylindre pour assurer la continuité de la surface intérieure de la chambre de travail. La présence de rainures transversales à l'avant et à l'arrière du volet, ou un défaut d'alignement vertical dû à un joint trop épais, pourrait déclencher une cavitation prématurée dans la section. Des ennuis de ce genre ont effectivement eu lieu, comme en témoignent les mesures du niveau sonore en cette section en l'absence de tout corps à expérimenter. Celte cavitation particulière a prouvé également l'existence de deux régimes d'écoulement différents, puisqu'elle se présentait pour la valeur critique 0,3 du coefficient relatif du tunnel lors de vitesses inférieures à $45 \mathrm{fps}(13,7 \mathrm{~m} / \mathrm{s})$, et pour la valeur 1,2 aux vitesses supérieures. Une vérification de l'alignement a alors montré que le volet dépassait de 0,050 pouce $(0,127 \mathrm{~cm})$. Après réparation du joint, la cavitation est apparue dans le tunnel pour un coefficient égal ou inférieur à 0,3 , quelle que soit la vitesse, laquelle a été poussée jusqu'à $80 \mathrm{fps}(24 \mathrm{~m} / \mathrm{s})$.

L'autre exemple de coexistence de deux régimes d'écoulement avec cavitation s'est présenté lors d'essais d'un corps à extrémité hémisphérique pourvue de clous à grosse tête. Ces clous, d'environ 1,8 pouces $(0,46 \mathrm{~cm})$ de long, 0,29 pouce $(0,7 \mathrm{~cm})$ de haut et 0,24 pouce $(0,6$ cm) de large, étaient fixés au corps de 4 pouces de diamètre $(10,2 \mathrm{~cm})$ immédiatement en arrière du point de tangence du cylindre et de son extrémité hémisphérique (ogive de calibre 0,5). Avec un certain nombre de clous on notait, à une vitesse déterminée, un brusque changement des conditions d'apparition de la cavitation. Les

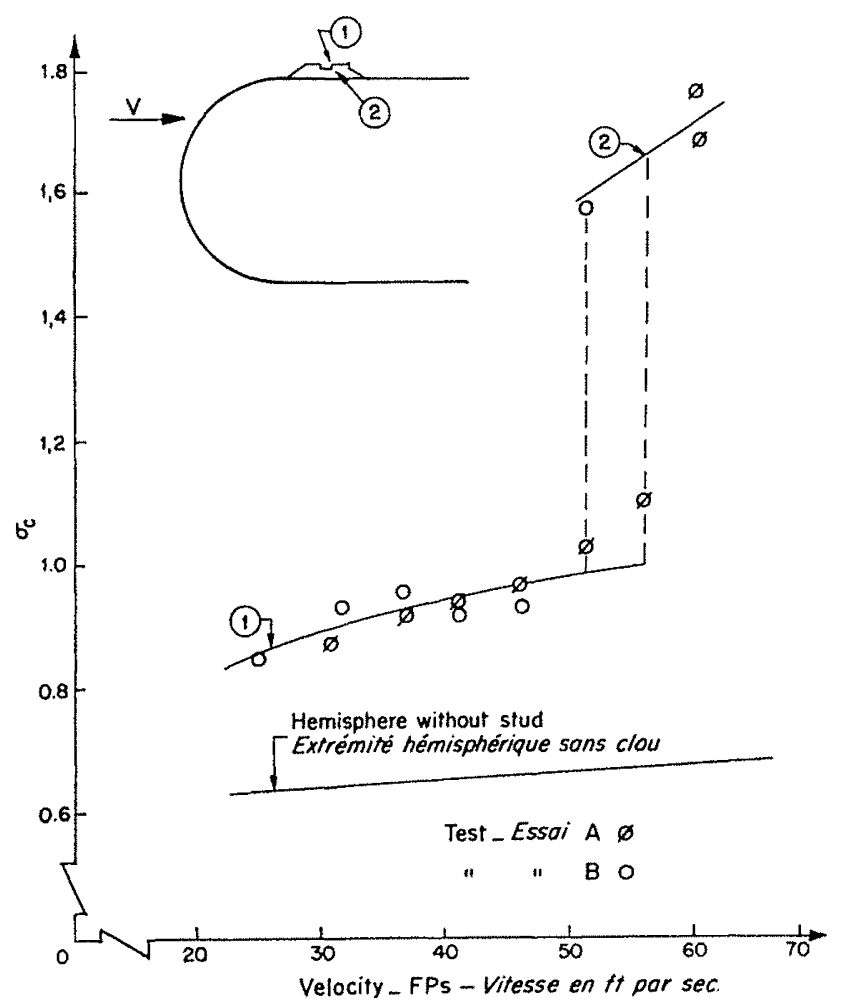

FIG. 10. - Manifestation de deux régimes d'apparition de la cavitation sur un clou.

Occurrence of two regimes of cavitation inception on a stud. 
valeurs obtenues avec un de ces clous sont portées sur la figure 10 sous forme d'un diagramme donnant $\sigma_{c}$ en fonction de la vitesse. Avec ce clou et pour des vitesses atteignant environ $50 \mathrm{fps}(15 \mathrm{~m} / \mathrm{s})$, la cavitation apparaissait dans le décollement de l'écoulement partant du sommet de l'entaille faite dans le clou. Aux vitesses plus grandes, la cavitation devenait instable et semblait se manifester au sein de l'écoulement en partant de l'angle inférieur aval de l'entaille. Avec d'autres clous, on notait des variations dans les conditions d'apparition de la cavitation pour des vitesses comprises entre 40 et 50 fps $(12$ et $15 \mathrm{~m} / \mathrm{s})$. Il serait intéressant d'étendre cette étude au cas de clous plus grands; mais déjà les quelques essais effectués semblent établir indiscutablement l'existence de deux régimes d'apparition de la cavitation, à des coefficients critiques nettement différents.

\section{Commentaires}

Si les études concernant les clous n'avaient été entreprises qu'à des vitesses inférieures à $50 \mathrm{fps}(15 \mathrm{~m} / \mathrm{s})$, on n'aurait noté qu'un seul genre de cavitation et une seule condition d'apparition. De même si les nez de 1 pouce $(2,54$ $\mathrm{cm}$ ) de calibre $1 / 8$ n'avaient pas été expérimentés à des vitesses supérieures à $60 \mathrm{fps}(18$ $\mathrm{m} / \mathrm{s}$ ), ou si l'on n'avait pas essayé des extrémités de calibre $1 / 8$ de dimensions plus fortes, le second régime d'écoulement concernant ce corps particulier serait passé inaperç. Toutes les fois où l'on a pu distinguer les deux régimes, le coefficient critique de cavitation était bien plus fort aux vitesses supérieures qu'aux vitesses moindres. Les prévisions quant aux conditions de l'apparition de la cavitation établies à partir d'essais sur modèles pouvaient donc se révéler inexactes. De plus, les essais sur les pointes en ogive ont montré l'existence de deux lois de similitude distinctes, selon qu'il s'agissait de l'un ou l'autre genre de cavitation. Là aussi, la transposition des résultats d'essais de cavitation sur modèle peut n'être pas valable tant que l'on n'aura pas bien saisi le genre de cavitation auquel on a afiaire.

Même dans le cas simple de l'ogive de calibre $1 / 8$, il n'a pas été possible de déterminer les facteurs qui déterminent le genre de cavitation qui apparaît. Pour les pointes moins bien profilées, il y a moins de probabilités de rencontrer les deux types de cavitation; celui avec décollement se présentera presque certainement, comme on le voit sur la figure 6. Dans le cas de nez mieux profilés, au contraire, la probabilité d'une cavitation avec décollement semble bien lointaine, si le calibre est assez grand. Ainsi pour le calibre 1/4, par exemple, l'examen de la figure 6 semble montrer que les deux types de cavitation seraient possibles aux grandes valeurs de $V d^{1 / 2}$ coïncidant avec des nombres de Reynolds faibles, conditions qui pourraient être réalisées dans une eau à température relativement basse.

La présence des deux régimes d'écoulement avec cavitation a pu être observée aussi sur d'autres familles de corps, mais on ignore à peu près tout quant aux lois qui gouvernent la similitude des phénomènes. A ce point de vue, le présent mémoire constitue un avertissement pour ceux qui entreprendraient des essais de cavitation sur modèle et leur suggère qu'il peut y avoir plus d'un régime de cavitation et plus d'une loi de similitude valable. Il y aurait donc des cas où il faudrait étendre les dimensions et les vitesses d'expérimentation au domaine des vraies grandeurs pour se rendre compte des incertitudes que peuvent comporter les essais.

\section{Conclusion}

L'étude de l'apparition de la cavitation telle (u'elle est décrite dans ce qui précède établit de façon claire et certaine que pour certaines formes de corps il peut y avoir deux régimes de cavitation se traduisant par des coefficients critiques d'apparition tout à fait dissemblables. Rien que pour les extrémités en ogive il existe deux règles différentes de similitude en fonction des dimensions et de la vitesse; il faudra donc s'entourer de beaucoup de précautions pour interpréter les essais sur modèles et en particulier s'assurer que les expériences ont bien été exécutées selon le régime d'écoulement qui correspond aux conditions du prototype.

Au sujet du cylindre circulaire terminé en ogive, auquel nous avons accordé plus d'attention, des conclusions ayant un caractère quantitatif peuvent être tirées. Ainsi, pour des nez de calibre zéro, le coefficient d'apparition de la cavitation est uniquement fonction du nombre de Reynolds caractérisant l'essai, du moins jusqu'à la valeur $1,5 \times 10^{6}$ pour des nez de 3 pouces $(7,6 \mathrm{~cm})$. Avec les nez de calibre $1 / 8$, deux genres de cavitation se présentent: aux faibles vitesses et pour les petites dimensions, c'est le genre avec décollement, et le coefficient $\sigma_{c}$ varie alors avec le nombre de Reynolds comme pour les nez de calibre zéro; aux dimensions et vitesses supérieures, la cavitation apparaît pour un coefficient notablement plus élevé, semblant varier avec $V d^{1 / 2}$.

Entre ces deux régimes la distinction peut être définie grosso modo comme se manifestant pour des nombres de Reynolds compris entre $3 \times 10^{5}$ 
et $8 \times 10^{5}$ et pour les $\mathrm{V} d^{1 / 2}$ compris entre 10 et $20 \mathrm{ft}^{1,5} / \mathrm{s}$. Dans le cas d'ogives de calibre plus fort, c'est-à-dire plus effilées, l'apparition de la cavitation dépend surtout de $\mathrm{V} d^{1 / 2}$ faisant soupçonner l'influence du nombre de Weber.

Nous avons bien tenu compte, pendant nos essais, de la variation dans la teneur en air et en gaz présents dans l'eau, mais ces effets étaient négligeables et noyés dans la dispersion des résultats. L'influence éventuelle de la teneur en air sur l'éventualité du double régime d'écoulement constaté sur les ogives de calibre 1/8 n'était guère perceptible à cause de la dispersion entièrement fortuite des valeurs obtenues aux essais. Néanmoins, dans un cas, il a été noté que l'air pouvait affecter les résultats concernant le coefficient critique, car, sous forme de petites bulles, il venait masquer ou altérer l'ambiance aux environs de l'endroit où apparaissait le phénomène.
Moyennant certaines précautions, les moyens visuels et les moyens acoustiques de détermination de la cavitation conduisent au même coefficienl critique; par contre, la comparaison des résultats obtenus par ces procédés à ceux fournis par la méthode de la pression utilisée à lowa il y a quelques années, dévoile des différences notables. Les procédés visuels et acoustiques donnent, en effet, pour les calibres forts, des valeurs du $\sigma_{c}$ moindres que celles trouvées à Iowa.

\section{Remerciements}

Les auteurs remercient le Dr. B. R. Parkin et le personnel du Laboratoire Hydrodynamique du CIT pour le concours et l'aide qu'ils ont voulu leur prêter. La plupart des valeurs obtenues sur les hémisphères et sur les ogives de calibre 1,5 l'ont ćté de concert avec cette Institution [2].

\section{BIBLIOGRAPHIE}

[1] R. W. Kemmen, J. T. MaGraw, and B. P. Parkin: "Mechanism of Cavitation Inception and the Related Scale-Effects Problem", Trans. ASME, Vol. 77, 1955.

[2] B.R.PARKiN and J. W. HoLL: "Incipient-Cavitation Scaling Experiments for Hemispherical and 1.5 Caliber Ogive-Nosed Bodies", The Pennsylvania State University, Ordnance Research Laboratory, Report No. NOrd 7958264, May 1953.

[3] H. Rouse and J. S. McNown: "Cavitalion and Pressure Distribution Head Forms at Zero Angle of
Yaw, Bulletin 32, state Ciniversity of lonn Studies in Engineering, 1948.

[4] R. T. KNapr: "Nose Cavilation - Ogives and Spherogives", OSRD Report 6.1 sr 207-1906, California Institute of T'echnology, 1945.

[5] R. B. Power, J. M. Ronentson and D. Ross: Garfield Thomas Tunnel Operations, The Pennsyluania State University, Ordnance Laboratory, Report No. NOrd 7958-211, May 1951.

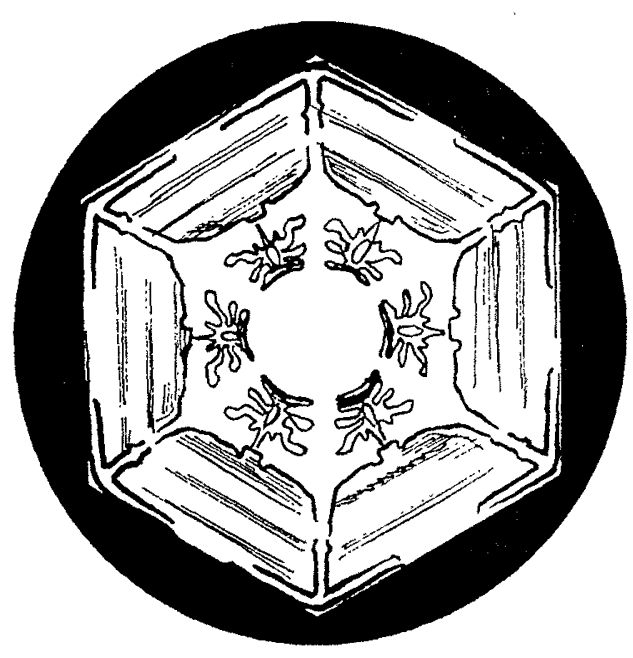




\title{
On several laws of cavitation scaling ${ }^{(*)}$
}

\author{
J.M. ROBERTSON (**), J.H. MCGINLEY (***) AND J.W. HOLL (****) \\ ORDNANGE RESEARCH LABORATORY, THE PENNSYLVANIA STATE UNIVERSTTY, UNIVERSITY PARK, PENNSYLVANIA
}

(See French text p. 540, for illustration)

Cavitation inception tests of ogive nosed circular cylinders of caliber between 0 and 1.5 verify that the primary factor determining the inception index is the curvature, or caliber. However, fluid properties and size and velocity conditions are found to introduce important secondary scaling effects. Inception on the finer noses is shown to scale primarily with the product of the velocity and the square root of the diameter while for the blunt shapes

\section{Introduction}

Many problems in fluid dynamics are studied at reduced scale in air tunnels, water tunnels, towing tanks, etc., because of the difficulties of full size studies. In interpreting the results of such model studies the similarity parameters relating the flows must be properly defined and the scaling laws determined for transferring results to prototype behavior. In the case of incompressible flows with friction, the Reynolds number is known to be the proper parameter and the boundary layer and drag expressions are related to it. In compressible flows the Mach number enters and in free surface liquid flows the Froude number is significant. For low speed liquid flows with a free surface, in which surface tension affects the flow, Weber number scaling enters. In the case of cavitating flows the situation is not yet clearly determined. The basic dimensionless parameter describing the nature of a flow relevant to cavitation is the cavitation index or number,

$$
\sigma=\frac{P-P_{V}}{1 / 2 \cdot V^{2}}
$$

$\left(^{*}\right)$ This work was supported by the U. S. Navy Bureau of Ordnance under contract NOrd 7958 .

$\left.\star^{\star \star}\right)$ Formerly Assistant Director in charge of W'ater Tunnel, now Professor of Theoretical and Applied Mechanies, University of Illinois.

(**) Formerly Research Associate, now with Haller, Raymond \& Brown, Inc., State College, Pennsylvania. $\left({ }^{* \star \star *}\right)$ Research Associate.
Reynolds number scaling is obtained. For intermediate curvatures as exemplified by the $1 / 8$ calibcr, either scaling law may apply and different inception indices can occur. The occurence of two regimes of cavitation inception on two other body shapes are also presented. Thus, not only may there be two laws for extrapolating model test results, but the two regimes of flow can have greatly different cavitution inception indices.

where $\mathrm{P}$ is the free stream absolute static pressure, $\mathrm{P}_{\mathrm{V}}$ is the vapor pressure of the liquid, the mass density and $V$ the free stream velocity, i.e., the relative velocity between the body involved and the undisturbed fluid. The value of this index for which cavitation first occurs is called the critical or inception index. In a cavitating flow the value of the index must be less than the inception value $\sigma_{c}$ of the particular body-the cavitation is more intense the lower $\sigma$ is below $\sigma_{c}$. In cavitation studies it is often assumed that $\sigma_{b}$, the inception index, is a function only of the body shape and orientation. Unfortunately other fluid conditions disturb this elementary assumption.

Recently Kermeen, McGraw, and Parkin [1] of the Hydrodynamics Laboratory of the California Institute of Technology, have reported studies of the inception of cavitation on simple axisymmetric bodies. These studies indicate different scaling laws for streamlined and bluff bodies. For streamlined shapes, 0.5 and 1.5 caliber ogive-nosed cylindrical bodies, the incipient cavitation index was found to increase with model size and free stream velocity and to approach in magnitude the minimum pressure coefficient. For sharp-edged discs, an extreme type of bluff body, the incipient cavitation index appeared to scale with Reynolds number. An analysis of the velocity and size variation for the streamlined bodies studied indicated dependence of the inception index on $\mathrm{V} d^{1 / 2}$ (velocity times square root of body diameter) suggesting a Weber number dependence [2]. This indicates a difference in cavitation scaling laws dependent upon the shape of the body. The question is, in a test 
of a model of a particular body or object, just which scaling law should be used in converting the results to prototype conditions. Recent cavitation studies, in the Ordnance Research Laboratory 48-inch Water Tunnel at The Pennsylvania State University, have resulted in a clearer insight into this question, although the complete picture is not yet available.

\section{Water tunnel tests}

In the ORL Water. Tunnel cavitation has been studied on dises and various nose shapes. For the extreme example of a bluff body a right circular cylinder parallel to the flow was used. This flatnosed cylinder can be considered a zero caliber ogive and hence included in the same geometric family as the more streamlined ogive shapes. The cavitation inception characteristics of the ogive family has been extensively studied [3], [4]. Figure 1 illustrates the contours of such noses. $\left(^{\star}\right)$ Ogive noses of $0,1 / 8,1 / 4$, and $1 / 2$ caliber and with diameters of $1 / 2,1,2,3$, and 4 inches have been studied in the 48 -inch throat water tunnel at speeds between 20 and $80 \mathrm{fps}$. In the tests reported in Reference [2], some of the data of which is included in the present analysis, $1 / 4$ to 8 inch noses of $1 / 2$ and $1-1 / 2$ caliber were tested in the tunnel.

In order to obtain repeatable results and eliminate a "hysteresis" effect, the same approach was used as developed at Caltech, to obtain the inception conditions [1], [2]. At a certain test velocity, the test pressure is lowered until cavitation is observed and then raised slowly until the cavitation just disappears. The disappearance of the last wisps of cavitation can usually be noted visually. In the present tests however most of the observations were made with a sensitive acoustic hydrophone system [5]. With this system, at ultrasonic frequencies, cavitation inception usually results in large changes in noise level. These are recorded on a sound level recorder with results such as shown in Figure 2. Clear definition of this inception pressure is apparent. In most cases visual observations of the cavitation at inception gave the critical pressure for inception to within 0.2 psi of that obtained acoustically. The minimum Water Tunnel pressure used in the tests was 5.5 psia. This results in a maximum possible error in the cavitation index of about 4 percent.

In evaluating the test results the cavitation index or number was determined in accordance with the relation given, with the vapor pressure determined from standard values based on the water temperature during the test. The Reynolds number, $\boldsymbol{K}=(\mathrm{V} d / \mathrm{v})$ was determined in terms of the diameter $d$ of the cylindrical body to which the noses were attached. The water temperature measured during the tests determined the kinematic viscosity $y$. Although Weber number dependency of cavitation

(*) The shapes of 0 to $1 / 2$ caliber are often termed plate-ogives. inception (implying surface tension effects) has been suggested, the surface tension variation obtainable with normal water is so small that this possibility could not be studied specifically. Therefore only the dimensional scaling factor $\mathrm{Vd}{ }^{1 / 2}$ is used.

\section{Inception on blunt noses}

The Caltech results on discs [1] indicate a strong Reynolds number dependency. As the cavitation observed on flat nosed cylinders is very similar to that observed on dises ( $\left.{ }^{*}\right)$ one would expect a Reynolds number dependency here too. That this occurs is shown by the data presented in Figure 3 for the 1/2, 1, 2, and 3 inch noses. Except for scatter, of the same order as that shown by the Caltech dise data, there is good agrecment with the trend curve for the discs. This zero caliber ogive data did not correlate with $\mathrm{V} d^{1 / 2}$ a plot on this basis giving separate lines for each size.

The small amount of data obtained for the cavitation inception on the 4 -inch zero-caliber ogive appears significantly different from that on the smaller sizes. It is thought that this may have been due to an air release phenomenon which disturbed some of the other tests particularly at the lower speeds. It was not feasible to test the large nose at high enough speeds to check on the variation in index and to ascertain what was occurring. The manner in which air release can influence a cavitation inception test was clearly noted in the case of a 1-inch 1/8-caliber ogive. Figure 4 shows the acoustic level readings for two successive runs at identical mean flow test conditions. The average air content was high and during the first run (Run 6) small air bubbles were observed in the flow approaching the nose. In this case no sharp delineation of cavitation inception occurrer, such as indicated by Figure 2. To remove these fine bubbles, the tunnel was run at a relatively high pressure for a few minutes and then the test rerun quickly before the bubbles could reappear. In this second run (Run 7) the normal type of cavitation inception occurred. As the total amount of air in the tumnel did not change during the test, it appears that its occurrence in fine bubbles in the one run masked the true point of inception. It is beyond the scope of this paper to speculate on which condition more truly represents conditions which occur in actual cavitating systems.

Some of the $1 / 8$ caliber ogive data indicated a bluff body behavior. The data from the 1,2, and 4 inch noses which does show this is also plotted in Figure 3 versus Peynolds number. Included also is one point from tests of a one inch nose at the University of Iowa [3]. Except for a number of low points from the one inch nose, the results for the $1 / 8$ caliber ogives indicate a Reynolds number variation similar to the zero caliber noses. As will be noted in later discussion, the nature of the flow occurring for this phenomenon was

(*) At least near inception. 
markedly different from that which occurred when the $V d^{1 / 2}$ type of scaling appeared to apply.

Also the magnitude of the inception index was significantly less than that of the other type of flow in which $\mathrm{Vd}^{1 / 2}$ scaling applied.

\section{Inception on streamlined noses}

As lhas been mentioned, the parameter $\mathrm{V} d d^{1 / 2}$ has been found to correlate cavitation inception on 0.5 and 1.5 caliber ogives [2]. In Figure 5 the ORL inception data for these two shapes is plotted as a function of $\mathrm{V} d^{1 / 2}$. In addition to the ORL data from Reference [2] for $1 / 2$ to 8 inch noses one set of measurements from more recent tests on a one inch 0.5 caliber nose is also included. The agreement with $\mathrm{V} d^{1 / 2}$ scaling noted in Reference [2] is apparent. In that reference it was also shown that correlation on the basis of Reynolds number was poor and that Caltech data (not shown in Figure 5) on $1 / 4$ to 4 -inch noses of these two calibers agreed closely with the ORL data. In fact the maximum divergence of the trend lines representing the data from two tunnels was about 0.02 in the index at $V d^{1 / 2}$ of about 20 . It has been noted for these two shapes $(0.5$ and 1.5 caliber $)$ that the inception index is below the minimum pressure coefficient of the body but approaches this value as the size and velocity increase [1], [2].

The available results of cavitation inception studies of the blunter ogives $(1 / 4$ and $1 / 8$ caliber) are also presented in Figure 5 . For the $1 / 4$ caliber only the one inch version was studied so that no verification of $\mathrm{Vd}^{1 / 2}$ scaling is possible. For certain of the test results on the $1 / 8$ caliber ogive $\mathrm{V} d^{1 / 2}$ scaling is apparent in Figure 5. Although the scatter in the data is much greater than for the fine ogives the 1,2 , and 4 inch sizes agree reasonably well.

\section{Effect of caliber on index}

The tests at the State University of Iowa [3] reported by Rouse and McNown in 1948 indicate a unique relation between the inception index and caliber for such rounded noses. The Iowa tests were of one inch noses at speeds up to $35 \mathrm{fps}$. A pressure distribution method was used to define the inception. In this method advantage is taken of the fact that cavitation modifies the surface pressure distribution (plotted in coefficient form) about the nose. The inception index $\sigma_{c}$ was chosen as that for which the initial disturbance of the pressure distribution was apparent. The Iowa data relating the inception index with caliber has been used as a standard in many hydroballistic designs. However, there has always remained a question as to whether other criteria (such as visual or acoustic) for inception might not result in significantly different values of inception indices. This uncertainty may result in the use of large "safety factors" in design or in unsatisfactory designs. The Iowa publication also includes a presentation of some Caltech data. [4] obtained by visual observations of the initial vapor pocket formation which at calibers less than 2 indicated appreciably lower values of $\sigma_{\sigma}$.

The results of the present tests permit comparison of the inception conditions obtained acoustically with those obtained by the pressure method. Such comparison of $\sigma_{c}$ as a function of caliber is shown in Figure 6. The Iowa results are presented as a single curve. Four different curves are shown for the present results. For cases in which $\mathrm{V} d^{1 / 2}$ scaling applies curves are shown for values of 10 and 30 . These particular values are shown because, as is evident in Figure 5, the value of $\sigma_{c}$ increases very slowly above $\mathrm{Vd} d^{1 / 2}$ of 30 , while the value of 10 corresponds to the lower limit of reliability of the tests. This value also corresponds to the upper limit of the Iowa tests (one-inch noses at $35 \mathrm{fps}$ ). For the noses in which Reynolds number scaling applies, two curves are presented for $\mathcal{R}=3 \times 10^{5}$ and $6 \times 10^{5}$. Although it is not clear just at what Reynolds numbers the Iowa data for the blunt ogives was obtained, it is indicated that some of their tests were in the range of 2 to $6 \times 10^{5}$.

The primary effect of shape, i.e., caliber, in determining the inception cavitation index is shown by Figure 6. The secondary effect related to the two scaling parameters are seen to be significant also. For calibers above $1 / 3$ the pressure method of defining $\sigma_{c}$ results in high values of the inception cavitation index. On the other hand, for the smaller calibers cavitation noise will generally occur before enough cavitation exists to modify the pressure distribution. For the blunt shapes the inception index varies with Reynolds number and the lowa pressure data appears to be in agreement with the present studies.

An explanation for the high values of $\sigma_{c}$, indicated by the pressure method, has been indicated by Kermeen, McGraw, and Parkin [1]. They found cavitation to occur prematurely in the pressure taps due to the nature of the flow past the opening. Therefore, this method indicated when cavitation would start in these taps rather than when it would start on the body. The Caltech data based on visual determination of inception and reported in 1945 [4] lies between the Iowa and $\mathrm{V} d^{1 / 2}=30$ curves of Figure 6 for nose calibers between 0.5 and 2. For a caliber of 1 it approaches closest to the present acoustic data in agreeing with the $\mathrm{V} d^{1 / 2}=30$ curve. As mentioned earlier, in the present tests close agreement between visual and acoustical means for noting cavitation inception was obtained. Any differences which occur are probably the result of difficulties in lighting and observation for comparable sizes and velocities.

\section{Cavitation on $1 / 8$ caliber noses}

For the various nose shapes studied, Rouse and McNown [3] found unstable flow only about $1 / 8$ caliber noses and one ellipsoid. With these two shapes they note that "the flow tended alternately to follow the irrotational flow pattern and to form 
a small but definite pocket of separation. Both the shorter and longer forms became stable within or below the range of the tunnel, but for difrerent reasons. Flow past blunt and concave forms achieves a stable separation pattern beginning at the abrupt change in boundary while in longer forms the separation either does not occur or is eliminated by turbulence at comparatively low values of $\mathcal{R}$. For the unstable intermediate forms, as $\mathcal{R}$ increases the separation is reduced and the value of the minimum pressure is also reduced; a limiting value is achieved, but only at higher values of $\mathcal{R}$. [3a].

A similar instability for $1 / 8$ caliber noses was observed in the present tests as indicated by the two sets of inception points in Figure 6. The higher index generally occurred at higher speeds with the same type of cavitation near inception as found for the $1 / 4,1 / 2$ and 1.5 caliber noses. These lwo types of flow are shown in the photographs of Figure 7. In Figure $7 a$ is shown the separated flow and the surface cavitation is pictured in Figure $7 b$.

In the first case, cavitation inception occurs in the turbulent eddies in the separated flow region just as it does for the blunter zero caliber ogives. In the other type of inception, the cavitation appears as a fine ring of cavitation on the surface of the nose near the point of tangency between the ogive curve and the body to which it is attached.

In many of the tests it was possible to note visually the differences between the separated cavitating flow and the ring type of cavitating flow near inception. However, this was not true for all cases as many inception points appear to have been erroneously identified. The existence of the two scaling relations shown in Figures 3 and 5 for $1 / 8$ caliber noses was well established by those tests in which there was no doubt in the identification of the type of caritation involved. These cases indicated that with the ring type of cavitation $\sigma_{c}$ was between 2.0 and 2.2 , while the separation type resulted in values less than 1.9. Therefore, the value of the cavitation inception index was used as the final criterion of which type of cavitation occurred.

In a number of tests the transition between the two types of flow was apparent between runs at different speeds and in a few cases both types of inception occurred in the same run and at the same speed. As the pressure was raised the separation cavitation would disappear giving its inception index and then the ring type cavitation would appear, due probably to some minor flow fluctuation, and the pressure would have to be raised even higher to obtain its inception condition. Some of the tests for the inception index at different speeds are shown in Figure 8 for the 1,2 and 4 inch noses. Three examples of the occurrence of both types of cavitation for the same test conditions are indicated by the solid vertical lines on this plot.

Following the suggestion by Rouse and McNown [3], it was thought that the demarkation between

(3 a) H. Rouse and J. S. MaNown (3) p. 13. the two types of flow condition would be on the basis of Reynolds number. A considerable number of tests were run on the one-inch noses at water tempcratures between 77-107 degrees Falurenheit in an attempt to clarify this point. The air content of the water was not held under strict control, and it varied from about 2 to 14 parts par million during this sroup of tests. Also in some of the tests, only one type of flow was found-in some cases the test was not carried to a high enough velocity and in others not to a low enough velocity. The results of this phase of the study are indicated in Figure 9. In many series of test runs only one type of cavitation was found. Assuming that the ring type of cavitation occurs at a higher speed than the separation type, the arrow on the symbols points in the direction at the expected occurrence of the other type of cavitation. All that can be concluded from this study is that the demarkation between the ring and separation type of cavitation on $1 / 8$ caliber ogives occurs for Reynolds numbers between 3 and $8 \times 10^{5}$ and values of the $V d^{1 / 2}$ parameter between 10 and 20 . Whether air content affects this cannot be said. Before terminating this discussion, it should be noted that the curve for $\sigma_{r}$. versus $\mathcal{R}$ approaches the value of $\sigma_{c}$ for the ring type of cavitation at some value of Reynolds number above $10^{6}$, above which the distinction between the two types is probably academic.

\section{Occurrence of two regimes on other bodies}

Besides the $1 / 8$ caliber ogive nose and the $1 / 2: 1$ ellipsoidal nose studied by Rouse and McNown, evidence of two regimes of cavitation flow and hence two different scaling laws for the same body has been noted for two more shapes tested in the ORL water tunnel.

The first of these involved part of the tunnel itself. Access to the four foot diameter working section of this tumnel is through a removable portion of the upper part of the cylindrical section [5]. This hatch cover of course should seat properly so that its surface represents a continuation of the interior surface of the working section. Transverse slots at the front and back of the hatch and any vertical misalignment due to too thick a gasket could be the cause of premature cavitation in the working section. That such trouble was occurring was evidenced by measurements of the acoustic noise level at the working section with no test bodies in it. This cavitation also indicated the existence of two regimes of flow, for it resulted in a critical tunnel index 0.3 at speeds below $45 \mathrm{fps}$ and an index of 1.2 above that speed. A check on the alignment of the hatch cover indicated that it was $0.050 \mathrm{in}$. too high. The gasket responsible for this has since been reworked, resulting in a cavitation inception index for the working section of 0.3 or below at all speeds up to $80 \mathrm{fps}$.

The other example of two regimes of cavitation flow or inception was found in the course of tests on studs attached to a hemispherical nosed body. These studs, about 1.8 inches long, 0.29 inches high 
by 0.24 inches wide, were attached to the fourinch diameter body just aft of the point of tangency between the hemispherical nose ( 0.5 caliber ogive) and the circular cylinder to which it was attached. With a number of the stud shapes a sudden change in the cavitation inception conditions was noted at a certain speed. Results for one stud are shown in Figure 10 as a plot of $\sigma_{c}$ versus the test speed. With this stud at speeds up to near $50 \mathrm{fps}$ the cavitation had its inception in the separated flow leaving the top of the slot in the stud. At higher speeds the cavitation was unstable and appeared to be occurring in the flow leaving the lower downstream corner of the slot. For other studs changes in the cavitation inception condition were noted at speeds between 40 and 50 fps. Although it would have been desirable to study the different flow conditions on larger studs, these studies are seen to definitely indicate two cavitation inception regimes with markedly different indices.

\section{Discussion}

If the stud studies just illustrated had not been run at speeds above $50 \mathrm{fps}$, only one type of cavitation inception condition would have been observed. Similarly if the 1 -inch $1 / 8$ caliber ogive noses had not been run at speeds above $60 \mathrm{fps}$ or the larger sized $1 / 8$ caliber noses tested the second flow regime for this shape would not have been observed. In all cases in which the two regimes have been found the inception cavitation index characterising the condition at the higher velocity has been significantly larger than that for the lower speeds. Hence prediction of expected conditions from model tests could be very misleading.

Furthermore, the ogive nose tests indicated the existence of two different scaling laws for the two types of cavitation. Velocity and size scaling of model cavitation tests results can thus also be in error if the proper type of cavitation is not identified.

Even for the simple case of the $1 / 8$ caliber ogive it has not been possible to determine the factors which determine which type of cavitation will occur. For blunter noses there is less likelihood of two types of cavitation; the separation type appears almost certain to occur as indicated in Figure 6. In the case of more streamlined nose shapes the occurrence of the separation type of cavitation appears remote for a sufficiently high caliber. For the $1 / 4$ caliber nose for instance, a study of Figure 6 suggests that two types of cavitation are possible at high values of $\mathrm{V} d^{1 / 2}$ and simultaneously low values of Reynolds number. A relatively low temperature water test would tend to produce this. For other families of body shapes the occurrence of two cavitation flow regimes has been observed, but little or nothing is known about the possible laws for scaling results. From that standpoint this article can but warn those using cavitation model tests that more than one regime and scaling law is possible. It may be necessary to run the tests at sizes and speeds corresponding to the full scale region to evaluate the seriousness of these uncertainties.

\section{Conclusions}

The studies of cavitation inception reported here indicate clearly and definitely that for some body shapes two possible cavitation regimes may occur. These regimes result in inception indices that can be quite different. Ogive noses, at least, follow different laws for size and speed scaling so that great care must be exercised in interpreting model test results. Testing must be conducted in the flow regime corresponding to the prototype conditions.

In the case of the ogive nosed circular cylinder to which major attention has been devoted in this article somewhat more quantitative conclusions can be drawn. For zero caliber noses the cavitation inception index is a unique function of the test Reynolds number, at least up to $1.5 \times 10^{6}$ for threeinch noses. For 1/8 caliber noses two types of cavitation inception occur-for lower velocities and sizes a separation type occurs in which $\sigma_{\text {r. }}$ scales with Reynolds number as in the case of the zero caliber uoses. For the larger sizes and velocities a cavitation occurs of significantly higher index which appears to scale with $\mathrm{V} d^{1 / 2}$. The demarkation between these two regimes is roughly defined as occurring in the range of Reynolds numbers between 3 and $8 \times 10^{5}$ and values of the parameter $\mathrm{Vd} d^{1 / 2}$ between 10 and $20 \mathrm{ft} .1 .5 / \mathrm{s}$. For ogives of larger caliber, i.c., finer noses, cavitation inception scales primarily according to $\mathrm{V} d^{1 / 2}$ suggesting a Weber's number effect.

Although variation in air or gas content of the water was introduced into the tests, the effects were generally so small as to be masked by the scatter in the test results. Possible effects of air content on the occurrence of the two flow regimes noted for the $1 / 8$ caliber ogive noses was unobservable due to the random scatter of the test results. As shown for one instance, however, air can greatly affect cavitation inception tests. In this case air in the form of fine bubbles masked or otherwise modified the occurrences near cavitation inception.

If proper care is taken, visual and acoustic means of determining cavitation inception conditions give essentially the same index. Comparison of the results by these methods with the pressure method used at Iowa a number of years ago indicates significant differences. For the larger calibers, lower values of $\sigma_{c}$ are found visually and acoustically than were found at Iowa.

\section{Acknowledgments}

The writers wish to acknowledge the cooperation and help received from Dr. B. R. Parkin and other members of the Hydrodynamics Laboratory of the California Institute of Technology. Most of the hemisphere and 1.5 caliber ogive test data was obtained in a cooperative program [2] with that institution. 